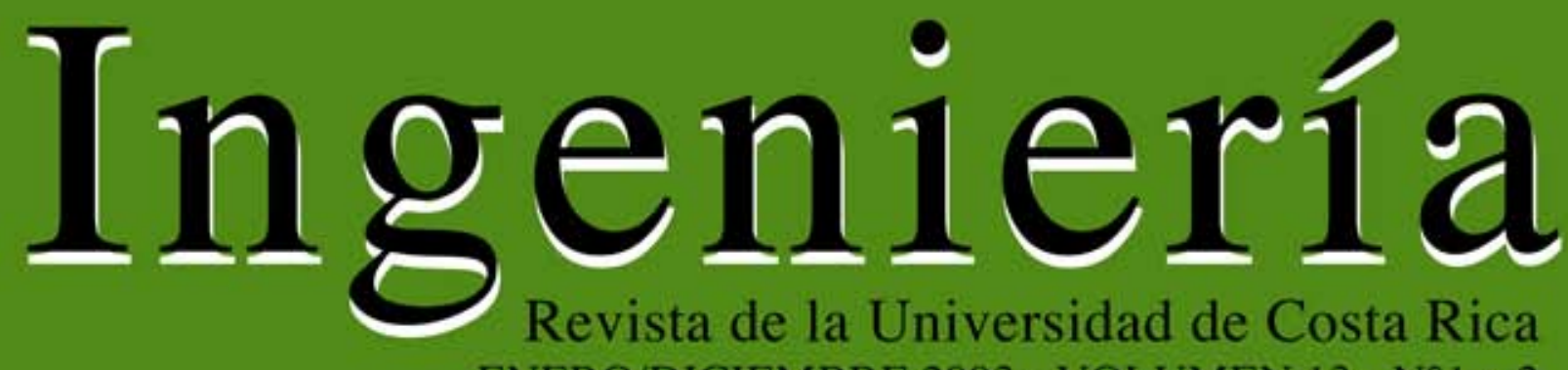
ENERO/DICIEMBRE 2003 - VOLUMEN 13 - N¹ y 2

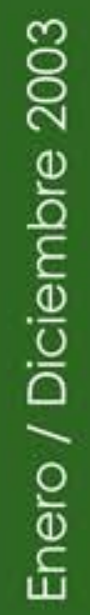

$\frac{m}{5}$
$\frac{c}{d}$
$\frac{5}{5}$
$\frac{\partial}{\circ}$

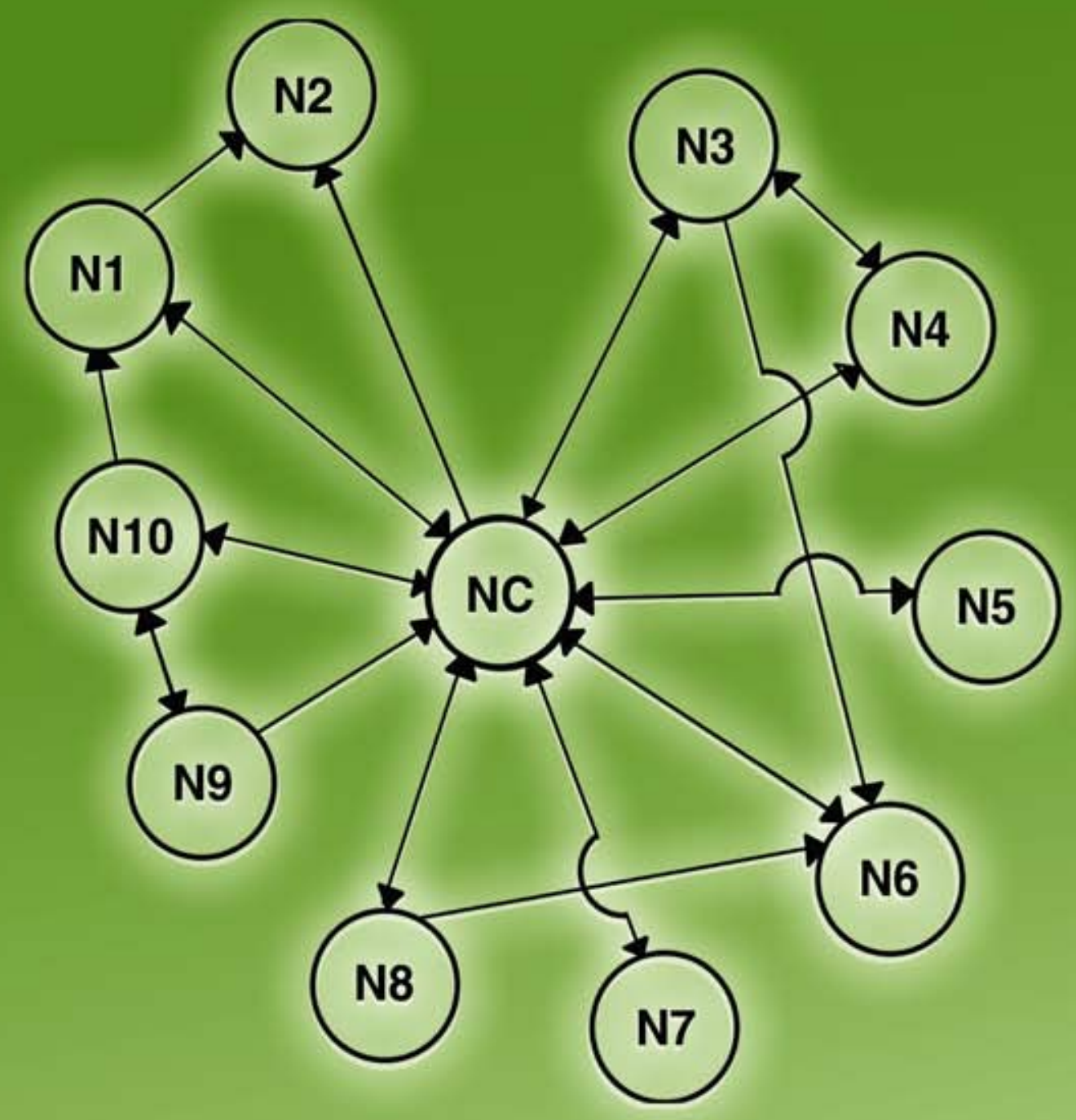

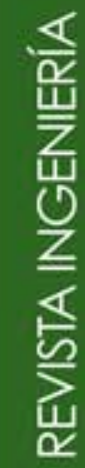




\title{
LAS LEYES DE KIRCHHOFF Y LAS REDES ELÉCTRICAS DE CORRIENTE CONTINUA
}

\author{
Rodolfo Herrera Jiménez.
}

\begin{abstract}
Resumen
En redes eléctricas de corriente continua las leyes de Kirchhoff son utilizadas para resolver el problema de la distribución real de la corriente y por tanto el estado de la red. En este artículo se demuestra rigurosamente, que conociendo las resistencias y las fuerzas electromotrices en la red, esas leyes son suficientes para determinar la distribución buscada de las corrientes. La prueba matemática desarrollada puede ser útil para fines de enseñanza.
\end{abstract}

Palabras clave: Redes eléctricas, leyes Kirchhoff, prueba matemática.

\begin{abstract}
In the continuous current electrical networks the Kirchhoff laws are used to solve the problem of the real distribution of current intensity and then the state of the network. It is rigorously proved in this article that knowing the values of the resistances and electromotive forces, those laws are sufficient to determine the network current distribution. The mathematical proof may be useful for teaching purposes.
\end{abstract}

Keywords: Kirchhoff laws, mathematical proof, networks electrical.

\section{DEFINICIONES Y PLANTEAMIENTO DEL PROBLEMA.}

Definición 1. Se llama red a un sistema finito de componentes orientadas c, en este caso "alambres", que se conectan en ciertos puntos $A_{l}, A_{2}, \ldots, A_{n}$ llamados nudos.

Se supone que la red es conexa, es decir, que dados dos nudos $A, B$ cualesquiera, se puede pasar de uno a otro mediante una sucesión finita de alambres $A B_{l}, B_{l} B_{2}, \ldots, B_{p} B$. Es claro que se puede suponer aquí que todos los nudos $A, B_{l}, B_{2}, \ldots, B_{p}, B$ son distintos.

Además se usarán las siguientes notaciones. Sea $k=1,2, \ldots, c$ y ${ }_{k}$ el alambre con una resistencia $r_{k}$ y llevando una fuerza electromotriz $e_{k}$, donde $e_{k}$ es un número positivo, negativo o nulo igual a la suma de los saltos del potencial al atravesar $k_{k}$ en el sentido positivo del alambre; ${ }_{k}$ es recorrido por la corriente (de intensidad) $i_{k}, i_{k}>0$ o $i_{k}<0$, según que ella fluya en el sentido positivo de $k$ o en el sentido opuesto; $i_{k}=0$ si no hay corriente en $k$.

Problema 1. Se trata de determinar $i_{k}$ conociendo $\operatorname{los} r_{k}, e_{k}$

Definición 2. Se llama 1-cadena una expresión simbólica de la forma: ${ }_{k=1}^{c} a_{k}$, donde las $a_{k}$ son números reales arbitrarios.

Definición 3. Las operaciones con 1-cadenas se definen según las fórmulas siguientes:

(i) La igualdad de 1-cadenas:

$$
{ }_{k=1}^{c} a_{k} \quad{ }_{k}={ }_{k=1}^{c} a_{k}^{\prime} \quad{ }_{k} a_{k}=a_{k}^{\prime} k=1, \ldots, c
$$

(ii) La adición de 1-cadenas:

$$
{ }_{k=1}^{c} a_{k} k^{+}{ }_{k=1}^{c} a_{k}^{\prime} \quad{ }_{k}={ }_{k=1}^{c}\left(a_{k^{+}} a_{k}^{\prime}\right)_{k}
$$


(i) La multiplicación de una 1-cadena por un número real:

$m\left({ }_{k=1}^{c} a_{k}{ }_{k}\right)={ }_{k=1}^{c}\left(m a_{k}\right)_{k}$

Teorema 1. Las 1-cadenas constituyen un espacio vectorial $C^{l}$ de dimensión $c$ donde c son una base para este espacio.

Teorema. Si la corriente que fluye en cada ${ }_{k}$ es $i_{k}$, entonces la 1-cadena:

$J={ }_{k=1}^{c} i_{k} \quad k$

suministra la distribución buscada de corrientes en la red.

Problema 2. El problema 1 consiste ahora en determinar la 1 -cadena en $J$.

Definición 4. Se llama 0 -cadena una expresión simbólica de la forma:

$$
{ }_{k=1}^{n} b_{k} A_{k}
$$

donde las ${ }_{l} b_{k}$ son números reales arbitrarios.

Análogamente como en la definición 2, se describen las operaciones de igualdad y adición de dos 0-cadenas y la multiplicación por un número real de una 0 -cadena. Por tanto:

Teorema 2. Las O-cadenas constituyen un espacio vectorial $C^{0}$ de dimensión $n$, donde $A_{1}, \ldots, A_{n}$ son una base para este espacio.

\subsection{Borde de una 1-cadena}

Definición 5. $\mathrm{Si}=A B$ es uno de los alambres orientados ${ }_{k}$ ( $A, B$ nudos), designamos por $\mathrm{y}$ llamamos borde de a la O-cadena siguiente:

$$
=B-A
$$

$\mathrm{Si}={ }_{k=1}^{c} a_{k k}$ es una 1-cadena arbitraria, el borde de es por definición:

$$
={ }_{k=1}^{c} a_{k k}
$$

La operación es una aplicación lineal de $C^{l}$ en $C^{0}$

Definición 6. Se llama ciclo cada 1-cadena cuyo borde es nulo.

Teorema 3. Los ciclos forman un subespacio $Z^{l}$ de $C^{l}$, el espacio nulo de . Los bordes constituyen un subespacio $B^{0}$ de $C^{0}$, el espacio imagen de .

Definición 7. Se llama malla una cadena de la forma $B_{1} B_{2}+B_{2} B_{3}+\ldots+B_{p} B_{1}$ donde $B_{1}, B_{2}, \ldots, B_{p}$ son nudos distintos.

Corolario 1. Una malla es por lo tanto un caso particular de un ciclo de forma: ${ }_{k=1}^{c} k$, donde cada ${ }_{k}$ es uno de los números $+1,-1,0$ con $k=1,2, \ldots, c$.

\subsection{Leyes de Kirchhoff}

(i) Sea $J={ }_{k=1}^{c} i_{k k}$ la 1 -cadena que define la distribución de las corrientes en la red. Entonces: $J=0$, es decir, $J$ es un ciclo, o sea que $\mathrm{J} \quad \mathrm{Z}^{l}$;

(ii) $\mathrm{Si}={ }_{k=1}^{c} \quad k \quad k$ es una malla cualquiera, entonces:

${ }_{k=1}^{c}{ }_{k} i_{k} r_{k}={ }_{k=1}^{c} e_{k}$

La $1^{\text {a }}$ ley expresa el hecho de que no hay acumulación de cargas en ningún punto de la red. La $2^{\mathrm{a}}$ ley se deduce de la ley de Ohm por un razonamiento elemental conocido.

\section{ESTUDIO DEL ESPACIO DE LOS CICLOS.}

El objeto de este párrafo es demostrar que cada ciclo puede expresarse, únicamente como combinación lineal de $r=c-n+1$ mallas oportunamente escogidas o "mallas fundamentales" y por lo mismo reducir la búsqueda de las $c$ corrientes $\mathrm{i}_{k}$ a la de $r$ corrientes auxiliares o corrientes circulantes $I_{v}(v=1,2, \ldots, r)$. 
Definición 8. Se llama índice de Kronecker de una 0 -cadena arbitraria $A={ }_{k=1}^{n} b_{k} A_{k}$ al número $\mathrm{I}(\mathrm{A})={ }_{k=1}^{n} b_{k} A_{k}$.

Si $A, B$ son dos 0 -cadenas arbitrarias y $a, b$ dos números cualesquier, es claro que se verifica:

$I(a A+b B)=a I(A)+b I(B)$

Teorema 4. Una 0 -cadena es un borde si y solo si su índice de Kronecker es nulo.

\section{Demostración:}

(i) Para un solo alambre $=A B$, tenemos por tanto $=B-A$, por tanto $\mathrm{I}()=0 . \mathrm{Si}$ $D=\left(\begin{array}{c}{ }^{c}{ }_{k=1} a_{k} k\end{array}\right)={ }_{k=1}^{c} a_{k k}$, es un borde cualquier, resulta inmediatamente: $I(D)=$ ${ }_{k=1}^{c} a_{k} I\left({ }_{k}\right)=0$.

(ii) Recíprocamente sea $\mathrm{B}={ }_{k=1}^{n} b_{k} A_{k}$, una 0 cadena cuyo índice de Kronecker sea nulo o sea $I(B)={ }_{k=1}^{n} b_{k}$. Sea $A$ un nudo arbitrario escogido (uno de los $A_{k}$ ). Puesto que la red es conexa se puede conectar $A$ con cada $A_{k}$ mediante una 1-cadena:

$$
{ }_{k}=A B_{1}+B_{1} B_{2}+\ldots B_{p} A_{k}, k=1,2, \ldots, c
$$

Se deduce inmediatamente:

$$
{ }_{k}=A_{k}-A
$$

Obtenemos pues:

$$
\begin{aligned}
& n_{k=1}^{n} b_{k} \quad k={ }_{k=1}^{n} b_{k}\left(A_{k}-A\right) \\
&={ }_{k=1}^{n} b_{k} A_{k}-\left({ }_{k=1}^{n} b_{k}\right) A={ }_{k=1}^{n} b_{k} A_{k}=B
\end{aligned}
$$

Por $B$ tanto es un borde.

Teorema 5. El espacio de los bordes $B^{0}$ es de dimensión $n-1$

Demostración: Por el teorema precedente una 0 -cadena: $\mathrm{B}={ }_{k=1}^{n} b_{k} A_{k}$ es un borde si y sólo si $I(B)={ }_{k=1}^{n} b_{k}=0$. O sea que

$$
B=b_{2}\left(A_{2}-A_{1}\right)+b_{3}\left(A_{3}-A_{1}\right)+\ldots+b_{n}\left(A_{n}-A_{1}\right)
$$

y por lo tanto, $B \quad B^{0}$ si y sólo si $B$ es una combinación lineal de las $n$-1 0 -cadenas: $A_{2}$ $A_{l}, \ldots, A_{n}-A_{l}$. Siendo estas cadenas por supuesto linealmente independientes, el teorema queda demostrado.

Teorema 6. El espacio $Z$ de los ciclos es de dimensión $r=c-n+l$.

Demostración. $r=\operatorname{dim} Z$ es la nulidad de, por otra parte (teorema 5): $(n-1)=$ dimension $B^{0}=$ rango de . Por consiguiente (como es sabido del álgebra lineal) $c=$ rango + nulidad $=n-1+r$, sea $r=c-n+1$. Ahora nos falta mostrar que se puede siempre tomar por base en $Z$, a un conjunto de mallas. Se necesitan primero dos resultados sobre ciclos:

Definición 9. Una 1-cadena ${ }_{k=1}^{c} a_{k}{ }_{k}$ se llama conexa si los alambres ${ }_{k}$ que figuran en ella, efectivamente (es decir, con coeficientes no nulos) forman una red conexa.

Lema 1. Cada ciclo es una suma de ciclos conexos.

Demostración. Sea $\mathrm{r}={ }_{k=1}^{c} a_{k}{ }_{k}$ un ciclo. Los alambres que figuran en él, efectivamente se distribuyen en unas cuantas subredes separadas (es decir, un punto de una no se puede conectar con un punto de otra, como en la página primera de este artículo), cada una de ellas es conexa, o sea: $={ }_{1}+{ }_{2}+\ldots{ }_{p}$ donde ${ }_{1},{ }_{2}, \ldots,{ }_{p}$ son 1 -cadenas conexas mutuamente separadas. Basta mostrar que estas cadenas son ciclos. Como es un ciclo, resulta:

$$
=\quad={ }_{1}+{ }_{2}+\cdots \quad p
$$

Si en ${ }_{l}$ figura efectivamente un cierto nudo, él no podría ya figurar así en ninguno de los ${ }_{1},{ }_{2}, \ldots, \quad, \quad$, puesto que las cadenas ${ }_{1},{ }_{2}, \ldots, p$ son separadas y por lo tanto disyuntas. Resultaría pues contrariamente a la hipótesis. Hay que concluir que $=\mathrm{y}$ así mismo $\quad{ }_{2}=\ldots={ }_{p}=$

Definición 10. Sea una 1-cadena conexa $={ }_{k=1}^{c} a_{k}{ }_{k}$, se dice que uno de los alambres, sea ${ }_{q}$, desconecta si $a_{q}{ }_{q}$ ya no es una cadena conexa. 
Lema 2. Ningún alambre desconecta un ciclo conexo.

Demostración. Sea $={ }_{k=1}^{c} a_{k} \quad$ y se supone que un alambre ${ }_{q}$ desconecta. Se va a mostrar que entonces $a_{q}=0$, de lo que resultaría $\quad a_{q}==$ ciclo conexo, es decir, contradicción.

De hecho puesto que ${ }_{q}$ desconecta, se tiene $a_{q}{ }_{q}={ }_{1}+{ }_{2}$, donde ${ }_{1}, \quad 2$ son dos cadenas separadas. Al tomar los bordes siendo $=0$ se obtiene: $a_{q}={ }_{1}+{ }_{2}$ (i). Sea ${ }_{k}=A B(A, B$ nudos). Entonces uno de los extremos de ${ }_{k}$ por ej. $A$, pertenece a la subred definida por ${ }_{2}$. La relación (i) se escribe entonces:

$a_{k} A-\quad{ }_{l}=a_{k} B+\quad{ }_{2}$,

siendo separadas las dos subredes de que se trata, cada miembro de la última relación es nulo o sea:

$a_{k} A=\quad{ }_{1}$.

Pero se sabe que el índice de Kronecker de un borde es nulo. Por tanto:

$a_{k}=I\left(a_{k} A\right)=I\left({ }_{1}\right)=0$

Teorema 7. Existe una base del espacio $Z$ de ciclos constituida por $r=c-n+1$ mallas ${ }_{v}(v=1,2, \ldots, r)$.

Demostración. Puesto que la dimensión de $Z$ es $r$, basta mostrar que las mallas engendran este espacio, lo que quiere decir que cada ciclo es una combinación lineal de mallas. En virtud del lema 1, es suficiente mostrarlo para un ciclo conexo. Sea pues $={ }^{c}{ }_{k=1} a_{k}{ }_{k}$ un ciclo conexo. $\mathrm{Si}$ por ejemplo $a_{l} 0$, el alambre ${ }_{l}\left(\right.$ sea $\left._{l}=A B\right)$, no desconecta (lema 2). Por lo tanto existen nudos distintos $B_{1}, B_{2}, \ldots, B_{p}$, todos diferentes a $A$ y $B$, tales que los alambres figuran efectivamente en.

Sea la malla $=A B+B B_{1}+\ldots+B_{p} A$. Entonces ${ }_{l}=-a_{l}$ es un ciclo, ya que no contiene efectivamente a, y además que no contiene ningún alambre que ya no figurara así en .

Es decir, ${ }_{1}=-a_{1}$ contiene efectivamente menos alambres que . Si, no es nulo, se repite la operación sobre cada parte conexa de . Así tras un número finito de $s$ operaciones (substracciones de múltiplos de mallas oportunas) reduce a 0 , o sea:

$$
\begin{array}{lllllll}
-b_{1} & 1 & b_{2} & 2 & b_{A} & =0, \quad{ }_{1}, \ldots,{ }_{A} \text { malla } 1 ; \\
{ }_{I}= & b_{1}=a_{1} .
\end{array}
$$

Por tanto, $\quad=b_{1} \quad l_{1} b_{2} \quad b_{A} \quad b_{A}$ viene así expresado como combinación lineal de mallas.

\section{MALLAS FUNDAMENTALES Y CORRIENTES CONTINUAS.}

Sea $\quad 1 \quad 2 \quad r(r=c-n+1)$ un sistema de mallas linealmente independientes, que constituyen una base de $Z^{\prime}$. Se supone escogido este sistema una vez para siempre, estas se llaman mallas fundamentales. Sea

$$
={ }_{k=1}^{c} \quad k^{k}, \quad \text { con } \quad k v=1,-1,0, r=c-n+1
$$

En virtud de la $1^{a}$ ley de Kirchhoff, siendo la cadena $\mathrm{I}={ }^{c}{ }_{k=1} i_{k} \quad k$ un ciclo, existen $r$ números $\mathrm{I}_{v}, v=1,2, \ldots, r$ únicos tales que:

$\mathrm{I}={ }_{k=1}^{c} i_{k} \quad k={ }_{v=I}^{r} I_{v} \quad(* *)$

Los $r$ números $I_{v}$ se llaman corrientes circulantes. Interpretando físicamente la fórmula $(* *)$ se puede decir que la distribución de corrientes en la red se obtiene "superponiendo" las corrientes circulantes $I_{v}$ que fluyen en las mallas fundamentales ${ }_{v}$. Más precisamente al sustituir $\left.{ }^{*}\right)$ en $(* *)$ e igualar los coeficientes se obtiene:

$i_{k}={ }_{v=1}^{r}{ }_{k v} I_{v}, k=1,2, \ldots, c \quad(* * *)$

$\mathrm{La}$ fórmula anterior (***) suministra las corrientes reales $i_{k}$ como combinaciones lineales de corrientes circulantes $I_{v}$. 


\section{UTILIZACIÓN DE LA 2 ${ }^{\mathrm{a}}$ LEY DE KIRCHHOFF : SOLUCIÓN DEL PROBLEMA.}

Una distribución ficticia de corrientes en una red $I={ }_{k=1}^{c} i_{k} \quad k$ se llama admisible si ella satisface a la $1^{a}$ ley de Kirchhoff o sea si $I$ es un ciclo. Para definir una distribución admisible cualquiera se dan arbitrariamente las corrientes circulantes $I_{v}, v=1,2, \ldots, r$ y calcular las $i_{k}$ por la fórmula (***). Si $I={ }_{k=1}^{c} i_{k} \quad k$ es una distribución admisible y si cada $i_{k}$ sufre un incremento $i_{k}$, se llama tal variación admisible si la distribución ${ }_{k=1}^{r}\left(i_{k}+i_{k}\right)_{k}$ es también admisible. Para cada distribución admisible se define:

$$
\begin{array}{ll}
={ }_{k=1}^{c} r_{k} i_{k}^{l} & =\text { Potencia calorífica } \\
={ }_{k=1}^{c} e_{k} i_{k} & =\text { Potencia química }
\end{array}
$$

Teorema 8. Una distribución admisible de corrientes satisface a la $2^{a}$ ley de Kirchhoff si y sólo si para ella, la función es mínima (es decir, que para cada otra distribución admisible, esta función no es menor).

Demostración. (i) se supone que la distribución admisible $I={ }_{k=1}^{c} i_{k} \quad k$ proporciona el mínimo a. Sea $\quad=\begin{array}{ccc}c & k=1 \quad k & k \\ \end{array}$ ${ }_{k}=+1,-1,2$. Sea $h$ un número arbitrario. A cada corriente $i_{k}$ se asigna un incremento ${ }_{k} h$. Así el ciclo $I$ aumenta de $I=\mathrm{h}^{c}{ }_{k=1} \quad k_{k}=\mathrm{h}$. Siendo este incremento un ciclo, la variación considerada es admisible. Los incrementos correspondientes de y son:

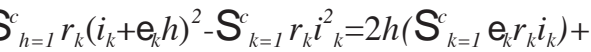

$$
\begin{aligned}
& +h^{2}\left(\begin{array}{cc}
c & \\
& 2 \\
k=1 & r_{k}
\end{array}\right) \\
& { }_{h=l}^{c} e_{k}\left(i_{k}+{ }_{k} h\right)-{ }_{k=1}^{c} e_{k} i_{k}=h{ }_{k=1 \quad k}^{c} e_{k}
\end{aligned}
$$

El incremento de es:

$$
h\left({ }_{k=1}^{c}{ }_{k} r_{k} i_{k^{-}}{ }^{c}{ }_{k=1}{ }_{k} e_{k}\right)+h^{2} \quad{ }_{k=1}^{c}{ }_{k}^{2} r_{k}
$$

Por hipótesis este incremento es no negativo, sea lo que sea el número $h$. Esto implica que el coeficiente de $h$ en la última expresión es nulo, o sea:

$$
{ }_{k=1}^{c}{ }_{k} r_{k} i_{k}={ }^{c}{ }_{k=1 \quad k} e_{k}
$$

Esto es exactamente la expresión de la $2^{a}$ ley de Kirchhoff para la malla arbitraria .

(ii) Se puede mostrar recíprocamente que si la $2^{a}$ ley de Kirchhoff viene satisfecha para una distribución admisible $I={ }_{k=1}^{c} i_{k \quad k}$ en todas las mallas entonces $I$ proporciona el mínimo a -2 . Se va a mostrar más, a saber, que si la $2^{a}$ ley de Kirchhoff viene satisfecha por tan solo en las mallas fundamentales ${ }_{v}, v=1,2, \ldots, r$ entonces -2 ya es mínima.

Una variación admisible arbitraria de corrientes puede obtenerse impartiendo incrementos arbitrarios a $h_{v}, v=1,2, \ldots, r$ a las corrientes circulantes $I_{v}, v=1,2, \ldots, r$. La variación correspondiente de las $i_{k}$ se calcula por la fórmula $(* * *)$ o sea:

$$
i_{k}={ }_{v=1}^{r} \quad k v h_{k}
$$

El incremento es:

$$
\begin{aligned}
& -\quad={ }_{k=1}^{c} r_{k}\left(i_{k}+{ }_{v=1}^{r}{ }_{k v} h_{v}\right)^{2}-{ }_{k=1}^{c} r_{k} i_{k}^{2} \\
& -2{ }_{k=1}^{c} e_{k}\left(i_{k}+{ }_{v=1}^{r}{ }_{k v} h_{v}\right)^{2}+2{ }_{k=1}^{c} e_{k} i_{k}= \\
& \text {-=2 } \quad{ }_{k, v}^{c, r} \quad k v h_{v} r_{k} i_{k}+1 / 2{ }_{k, v}^{c, r} r_{k}\left({ }_{k v} h_{v}\right)^{2}- \\
& { }_{v, k}^{c, r} \quad{ }_{k v} h_{v} e_{k}
\end{aligned}
$$

El coeficiente de $h_{v}, v=1,2, \ldots, r$ es:

$2{\stackrel{c}{c}{ }_{k=1} \quad k v} r_{k} i_{k^{-}}{ }_{k=1 \quad k v}^{c} e_{k}=0$

puesto que por hipótesis la $2^{a}$ ley de Kirchhoff viene satisfecha para cada malla fundamental ${ }_{v}$. Finalmente:

$\Delta(-2)=\quad{ }_{k, v}^{c, r} r_{k}\left({ }_{k v} h_{v}\right)^{2} \geq 0$

es decir, la distribución I proporciona el mínimo a -2 . 


\section{CONSECUENCIAS. SISTEMA LINEAL RESOLVENTE.}

Resulta de la última demostración que si una distribución admisible $I$ satisface a la $2^{a}$ ley de Kirchhoff tan solo en las $r$ mallas fundamentales ${ }_{v}$, ella satisface a la $2^{a}$ ley de Kirchhoff en todas las mallas.

Escribiendo la expresión de esta ley para cada una de las $r$ mallas fundamentales se obtiene $r=n-c+1$ ecuaciones lineales respecto a las desconocidas $i_{k}$. Sustituyendo $i_{k}$ por $(* * *)$ se consigue un sistema de ecuaciones lineales con $r$ desconocidas $I_{1}, I_{2}, \ldots, I_{r}$. Se llama a este sistema sistema resolvente.

Si sepuederesolversedeterminan inmediatamente las $i_{k}$ mediante (***) y el problema de hallar la distribución real de $i_{k}$ queda resuelto. Para mostrar que el sistema resolvente tiene una solución única, se escribe explícitamente un sistema equivalente, utilizando las expresiones $\mathrm{y}$ mediante las $I_{v}$. Así se tiene:

$$
={ }_{k=1}^{c} r_{k} i_{k}^{2}={ }_{k=1}^{c} r_{k}\left({ }_{v=1 \quad k v}^{r} I_{v}\right)^{2}
$$

esto es una forma cuadrática:

$$
={ }_{v=1}^{c} c_{v} I I_{v}, \mathrm{c}_{v}=\mathrm{c}_{v}
$$

Esta forma cuadrática es positiva definida. De hecho es claro que $\geq 0$, además $=0$ implica $i_{k}=0, k=1,2, \ldots, \mathrm{c}$, es decir:

$I={ }_{k=1}^{c} i_{k} \quad k=0$

Finalmente por $(* *) I_{v}=0, v=1,2, \ldots, r$, puesto que la expresión $(* *)$ es única.

(ii) $\quad={ }_{k, v}^{c, r} e_{k}\left({ }_{k v} I_{v}\right)={ }^{r}{ }_{v=l} E_{v} I_{v}$

donde

$E_{v}={ }^{c}{ }_{k=1} \quad k v e_{k}$

es la fuerza electromotriz total entre las mallas $I_{v}$.
Ahora un sistema equivalente al sistema resolvente, se establece escribiendo que es el mínimo para los valores buscados de los $I_{v}$.

Si a los $I_{v}$ se les asignan incrementos arbitrarios $h_{v}, v=1,2, \ldots, r$, el incremento correspondiente de es:

$$
\begin{aligned}
& \Delta()={ }^{r}{ }_{v} c_{v}(I+h)(I+h)-{ }^{r}{ }_{v} c_{v} I I_{v}- \\
& -2{ }^{r}{ }_{v} E_{v}\left(I+I_{v}\right)+2{ }^{r} E_{v} I_{v}= \\
& ={ }^{r}{ }_{v} c_{c v} I h_{v^{-}}{ }^{r}{ }_{v} I_{v} h_{\mathrm{v}}+{ }^{r}{ }_{v} c_{v} h h_{v}
\end{aligned}
$$

Esto debe ser mayor o igual a cero para cada elección de $\operatorname{los} h_{v}$, lo que equivale al hecho de que los coeficientes de todos los $h_{v}$ en la última expresión, deben ser nulos. Esto da el sistema siguiente:

$$
\begin{aligned}
& { }_{=l}^{r} c_{v} I=E_{v}, v=1,2, \ldots, r \quad \text { o sea } \\
& \underbrace{1 \quad}_{v}=E_{v}, v=1,2, \ldots, r
\end{aligned}
$$

que es el sistema buscado equivalente al sistema resolvente.

Teorema 9. El sistema (****) tiene una solución única.

Demostración. Hay que mostrar que $\operatorname{Det}\left(c_{i j}\right) \neq 0$ Para esto basta asegurarse que si $E_{V}=0, v=1,2, \ldots, r$, la única solución de (****) es $I_{v}=0, v=1,2, \ldots, r$.

Pero el problema se reduce entonces a determinar el mínimo de. Siendo una forma positiva definida, su mínimo igual a cero se realiza únicamente tomando $I_{v}=0, v=1,2, \ldots, r$ (generalmente se demuestra en álgebra lineal, que el determinante de una forma cuadrática positiva definida es siempre positivo). Con lo que queda demostrado el teorema.

El problema está ahora resuelto. La discusión hecha (en particular el último teorema) demuestra que las dos leyes de Kirchhoff, son suficientes para determinar la distribución real de las corrientes en la red, conociendo las resistencias $r_{k}$ y las fuerzas electromotrices $e_{k}$. 


\section{SOBRE EL AUTOR}

\section{Rodolfo Herrera Jiménez.}

Dr. Ing. prof. Emérito en ciencias cognoscitivas, Facultad de Ingeniería, Universidad de Costa Rica.

Teléfono: 253-4549

Correo electrónico: rodolfoh@racsa.co.cr
El tema de este artículo fue desarrollado en el curso sobre campo electromagnético que dictó el autor, para ingenieros electricistas y físicos en el año 1965, en la Facultad de Ingeniería de la Universidad de Costa Rica. Para los amigos del rigor matemático en este campo puede serles útil. 
